\title{
Conformational switching of FMN and THF riboswitches
}

\author{
J Stagno ${ }^{1}, \mathrm{H}$ Wilt $^{2}, \mathrm{~K} \mathrm{Tan}^{3}, \mathrm{P} \mathrm{Yu}{ }^{1}, \mathrm{Y} \mathrm{Wang}^{4}$ \\ ${ }^{1}$ National Cancer Institute, Frederick, MD, ${ }^{2}$ MCL, Frederick, MD, ${ }^{3}$ Argonne National Laboratory, \\ Argonne, IL, ${ }^{4}$ Structural Biophysics Laboratory, National Cancer Institute \\ Jason.stagno@nih.gov
}

Flavin mononucleotide (FMN) and tetrahydrofolate (THF) are essential cofactors for a number of biosynthetic pathways in bacteria. As such, the FMN and THF RNA riboswitches, which depend on those cofactors for genetic regulation, present attractive targets for developing novel antimicrobial therapeutics. Riboswitches get their names from the conformational switching that takes place upon ligand binding, which enables control of transcription or translation of the gene to which the riboswitch is associated. The first step in understanding the structural basis for the switching mechanism of a riboswitch requires structure determination of both ligand-free and ligand-bound conformational states. We recently determined novel structures of the FMN and THF riboswitches in the absence of their respective ligands, illustrating the conformational differences from their ligand-bound conformers. In the case of FMN riboswitch, the flexible L4 loop adjacent to the FMN-binding site alters the structure and stability of the regulatory helix P1, depending on whether or not FMN is bound. Similarly, the apo structure of THF riboswitch illustrates a different configuration of the ligand binding pocket that subsequently alters the conformations of the helices P1 and P3. Together, these structures increase our understanding of the ligand-dependent control mechanisms of RNA riboswitches. 